\title{
Frontal QRS-T Angle and the Incidence of Intracranial Cerebral Aneurysm
}

\author{
Efe Edem ${ }^{1 *}$, Cagin Senturk ${ }^{2}$ \\ ${ }^{1}$ Cardiology Department, Izmir Tinaztepe Hospital, Turkey \\ ${ }^{2}$ Interventional Radiology Department, Izmir Tinaztepe Hospital, Turkey
}

*Corresponding Author: Efe Edem, Cardiology Department, Izmir Tinaztepe Hospital, Turkey, Email:edemefe@gmail.com

\begin{abstract}
Background: The purpose of the present study was to analyze the relationship between frontal plane QRS-T angle on electrocardiogram (ECG) and the incidence of intracranial cerebral aneurysm (ICA).

Methods: 54 patients who underwent cerebral angiography (CA) between October 2013 and January 2017 were enrolled in the study. Patients included in the study were separated into two groups according to the existence of ICA on CA. Group One consisted of patients with normal findings in CA (n:27) and Group Two consisted of patients diagnosed with ICA (n:27). Two groups were compared in terms of frontal plane QRS-T angle on ECG by using hospital records.
\end{abstract}

Results: Group Two consisted of more hypertensive patients and this indicated a significant difference in terms of existence of hypertension between two groups $(p=0.026)$. Smoking habbit was more common in Group Two and this indicated a statistically significant difference ( $p<0.001)$. 12-lead ECG of patients in Group Two tended to demonstrate a wider frontal plane QRS-T angle compared to patients in Group One $(p<0.001)$.

Conclusion: A wide frontal plane QRS-T angle appears to be associated with the existence of ICA and interpretion of a simple 12-lead ECG may help us to establish patients with ICA on patients presenting with neurological symptoms and/or subarachnoid hemorrhage.

Keywords:Electrocardiography, Cerebral Aneurysm, Cerebral Artery Diseases

\section{INTRODUCTION}

QRS-T angle is a novel marker of ventricular repolarization heterogeneity. It is described as the angle between the main electrical axes of ventricular depolarization and repolarization [1]. Previous studies in literature showed that hypertension and coronary artery disease (CAD) may cause a widened QRS-T angle on electrocardiogram (ECG) [2-5].

An intracranial cerebral aneurysm (ICA) is a bulging, weakened area in the wall of an artery in the brain and these areas have a potential danger of bursting and intracranial bleeding. There are many risk factors for development of ICA such as smoking, hypertension, family history of brain aneurysms, age over 40 , collagen tissue disorders, presence of an arteriovenous malformation, cocaine use, tumors and traumatic head injury. Since hypertension and smoking are common risk factor for CAD and intracranial cerebral aneurysms and CAD can cause an abnormal frontal plane QRS-T angle on ECG, we aimed to investigate whether the same relationship exists between ICA and frontal plane QRS-T angle in this study.

\section{Material AND Methods}

The current study is a single-center retrospective study. The study protocol was approved by a local noninvasive Ethics Committee. 63 patients who underwent cerebral angiography (CA) between October 2013 and January 2017 were screened. Patients who were previously diagnosed with coronary artery disease and/or congestive heart failure that may already exhibit widened frontal plane QRS-T angle were excluded from the study. Remaining 54 patients were enrolled in the study.

Diabetes mellitus (DM) was defined as having a HbA1C level $\geq 6.5 \%$ or fasting plasma glucose 
level $\geq 126 \mathrm{mg} / \mathrm{dl}$ during hospitalization or being currently on anti-diabetic treatment. Hypertension was defined as having a systolic blood pressure level at least $130 \mathrm{mmHg}$ and/or a diastolic blood pressure level at least $80 \mathrm{mmHg}$ on during hospitalization or currently using an anti-hypertensive treatment.

CA was indicated only when the patients had neurological symptoms and/or subarachnoid hemorrhage with a prediagnosis of ICA according to brain computerized tomography scanning. Patients included in the study were grouped into two groups according to the existence of ICA on CA. severity of PAD. Group One consisted of patients diagnosed with ICA (n:27) and Group Two consisted of patients with normal findings on CA ( $\mathrm{n}: 27)$.

\section{ElECTROCARDIOGRAPHIC EVALUATION}

The 12-lead ECG (Nihon Kohden, Tokyo, Japan) was recorded from all patients with a 25 $\mathrm{mm} / \mathrm{s}$ speed, $10 \mathrm{~mm} / \mathrm{mV}$ height, and $0.16-100$ $\mathrm{Hz}$ filter range at rest in the supine position. Frontal QRS and T wave axes were available in the reports of the ECG machine. QRS and T axes were checked from these reports, and the frontal QRS-T angle was calculated as the absolute value of the difference between QRS and $\mathrm{T}$ wave axes (QRS axis - $\mathrm{T}$ axis). If this difference exceeded $180^{\circ}$, the difference was calculated by subtracting from $360^{\circ}$.

\section{Statistical Analysis}

Statistical analysis was performed using IBM SPSS Statistics, Version 20.0 (IBM, Armonk, NY). Data were presented as mean, standard deviation, median, minimum, maximum, percent and number. Cross tables were constructed for qualitative variables and their distribution was assessed by Chi Square Test. A $p$ value of $<0.05$ was considered a statistically significant difference for the $95 \%$ confidence interval.

\section{Results}

The study group consisted of 54 patients. Mean age of the study population was $46.22 \pm 12.61$. 28 of them were male $(51.9 \%)$ and 26 of them were female $(48.1 \%)$. Mean frontal QRS-T angle was $68.04^{\circ} \pm 32.18^{\circ}$. Demographic characteristics of the study population is presented in Table 1. The localization of ICA was anterior cerebral artery in 14 patients, internal carotid artery in eight patients and middle cerebral artery in five patients. Group Two consisted of more hypertensive patients and this indicated a significant difference in terms of existence of hypertension between two groups $(\mathrm{p}=0.026)$. Smoking habbit was more common in group 2 and this indicated a statistically significant difference $(\mathrm{p}<0.001) .12-$ lead ECG of patients in Group Two tended to demonstrate a wider frontal QRS-T angle compared to patients in Group One $\left(85.30^{\circ} \pm\right.$ $32.42^{\circ}$ vs. $50.78^{\circ} \pm 21.01^{\circ}, \mathrm{p}<0.001$ ) (Figure 1).

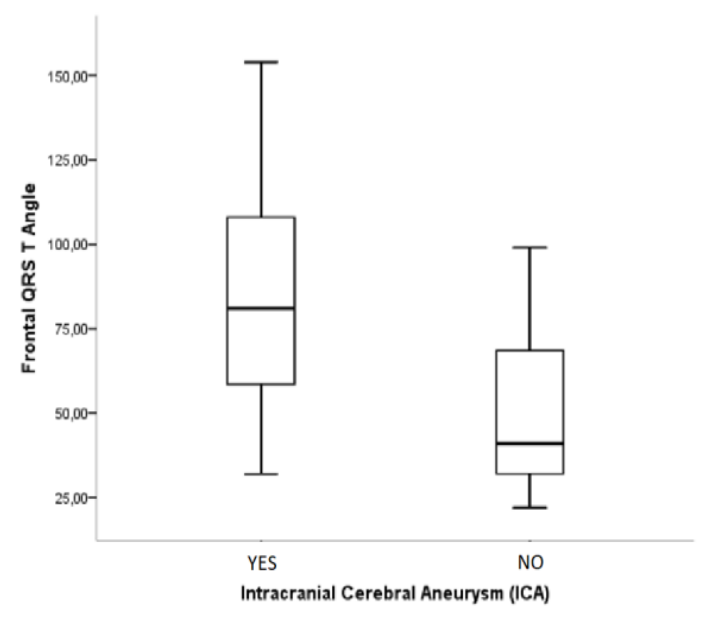

Figure1.Figure demonstrating the significant frontal plane QRS-T angle difference between two groups $(p<0.001)$

Table1. Demographic characteristics of the study group

\begin{tabular}{|c|c|c|c|}
\hline & $\begin{array}{l}\text { Group } 1 \\
(\mathrm{n}: 27)\end{array}$ & $\begin{array}{l}\text { Group } 2 \\
(\mathrm{n}: 27)\end{array}$ & $\begin{array}{l}\mathrm{P} \\
\text { Value }\end{array}$ \\
\hline Age (years) & $44.30 \pm 13.56$ & $48.15 \pm 11.51$ & $\mathrm{p}=0.222$ \\
\hline $\begin{array}{l}\text { Diabetes Mellitus } \\
\text { (positive vs. negative) }\end{array}$ & $\begin{array}{l}(\mathrm{n}: 4 \text { vs } n: 23) \\
14.8 \% \text { vs. } 85.2 \%\end{array}$ & $\begin{array}{l}\text { (n:5 vs n: } 22) \\
18.5 \% \text { vs. } 81.5 \%\end{array}$ & $\mathrm{p}=0.715$ \\
\hline $\begin{array}{l}\text { Hypertension } \\
\text { (positive vs. negative) }\end{array}$ & $\begin{array}{l}\text { (n:6 vs n: } 21) \\
22.2 \% \text { vs. } 77.8 \%\end{array}$ & $\begin{array}{l}\text { (n:15 vs } \mathrm{n}: 12) \\
55.6 \% \text { vs. } 44.4 \%\end{array}$ & $p=0.026$ \\
\hline $\begin{array}{l}\text { Smoking } \\
\text { (positive vs. negative) }\end{array}$ & $\begin{array}{l}\text { (n:7 vs n:20) } \\
25.9 \% \text { vs. } 74.1 \%\end{array}$ & $\begin{array}{l}\text { (n:21 vs n:6) } \\
77.8 \% \text { vs. } 22.2 \%\end{array}$ & $\mathrm{p}<\mathbf{0 . 0 0 1}$ \\
\hline $\begin{array}{l}\text { Gender } \\
\text { (Male vs. Female) }\end{array}$ & $\begin{array}{l}\text { (n:12 vs } \mathrm{n}: 15) \\
44.4 \% \text { vs. } 55.6 \%\end{array}$ & $\begin{array}{l}\text { (n:16 vs n: } 11) \\
59.3 \% \text { vs. } 40.7 \%\end{array}$ & $\mathrm{p}=0.414$ \\
\hline $\begin{array}{lll}\text { Frontal Plane } & \text { QRS-T } \\
\text { Angle } & & \\
\end{array}$ & $50.78^{\circ} \pm 21.01^{\circ}$ & $85.30^{\circ} \pm 32.42^{\circ}$ & $p<0.001$ \\
\hline
\end{tabular}




\section{DISCUSSION}

QRS-T angle, described as the angle between the mean QRS and $\mathrm{T}$ vectors, reflects the main orientation of electrical heart activity during ventricular depolarization and repolarization. A wider QRS-T angle indicates an abnormal set of ventricular repolarization and is considered as a novel risk marker for cardiovascular morbidity and mortality compared to other traditional cardiovascular risk factors and ECG risk parameters [2]. Recent studies demonstrated that hypertension and CAD were probably the main underlying pathophysiological conditions for an abnormal QRS-T angle [3-5] . Risk factors of CAD including smoking and hypertension are also known to contribute in the formation of ICA.

Indeed, our single center research indicated that a wide frontal plane QRS-T angle was associated with the existence of ICA. The relationship between frontal plane QRS-T angle and ICA might be explained by mutual risk factors of ICA and cardivascular diseases, which eventually deteriorate the axes of ventricular depolarization and repolarization.

\section{CONClusion}

Current study showed that a widened frontal QRS-T angle is related with the existence of ICA and a large frontal QRS-T angle on ECG may serve as a helpful and non-invasive marker for predicting the existence of ICA in patients presenting with neurological symptoms and/or subarachnoid hemorrhage. Further large-scale studies are required to emphasise the predictor role of frontal plane QRS-T angle in patients suffering form ICA.

\section{REFERENCES}

[1] Oehler A, Feldman T, Henrikson CA, Tereshchenko LG. QRS-T angle: a review. Ann Noninvasive Electrocardiol. 2014;19:534-42.

[2] Voulgari C, Pagoni S, Tesfaye S, et al: The spatial QRS-T angle: implications in clinical practice. Curr Cardiol Rev 2013; 9: 197-210.

[3] Dern PL, Pryor R, Walker SH, et al: Serial electrocardiographic changes in treated hypertensive patients with reference to voltage criteria, mean QRS vectors, and the QRS-T angle. Circulation 1967; 36: 823-829.

[4] Palaniswamy C, Singh T, Aronow WS, et al: A planar QRS-T angle $>90$ degrees is associated with multivessel coronary artery disease in patients undergoing coronary angiography. Med Sci Monit 2009; 15:MS31-MS34.

[5] Walsh JA, Soliman EZ, Ilkhanoff L, et al: Prognostic value of frontal QRS-T angle in patients without clinical evidence of cardiovascular disease (from the Multi-Ethnic Study of Atherosclerosis). Am J Cardiol 2013; 112: $1880-1884$.

Citation: Efe Edem, Cagin Senturk. Frontal QRS-T Angle and the Incidence of Intracranial Cerebral Aneurysm. ARC Journal of Cardiology. 2018; 4(1)12-14. doi: dx.doi.org/11.20431/2455-5991.0401004.

Copyright: (0) 2018Authors. This is an open-access article distributed under the terms of the Creative Commons Attribution License, which permits unrestricted use, distribution, and reproduction in any medium, provided the original author and source are credited. 